\title{
Heat- and Particle-Deposition Distribution on Helical Divertor Plates in LHD During Real-Time Magnetic-Axis Swing Operations
}

\author{
Hironori OGAWA, Suguru MASUZAKI ${ }^{1)}$, Tsuguhiro WATANABE ${ }^{1)}$, Tomohiro MORISAKI ${ }^{1)}$, \\ Takashi MUTOH $^{1)}$, Ryuhei KUMAZAWA ${ }^{1)}$, Tetsuo SEKI ${ }^{1)}$, Kenji SAITO ${ }^{1)}$, Yukio NAKAMURA ${ }^{1)}$, \\ Akio KOMORI ${ }^{1)}$, Nobuyoshi OHYABU ${ }^{1)}$ and the LHD experimental group \\ Graduate University for Advanced Studies, Hayama 240-0163, Japan \\ ${ }^{1)}$ National Institute for Fusion Science, Toki 509-5292, Japan
}

(Received 19 June 2007 / Accepted 2 August 2007)

\begin{abstract}
Investigations of heat- and particle-deposition distributions on helical divertor plates were performed both experimentally and numerically with the Large Helical Device (LHD). The distributions were measured by thermocouples and Langmuir probe arrays embedded in the divertor plates. They are similar to the distribution of field lines with long connection lengths, which are estimated numerically by field-line tracing calculations. Localized heat- and particle-deposition distributions were observed, and were found to be determined primarily by the magnetic-field line structure in the divertor region. They vary depending on the configuration - the intensively loaded areas move with a change of the magnetic configuration. Using this property, a swing of the magnetic axis successfully dispersed the heat and particle loads on the divertor plate during long-pulse discharges. The magnetic-axis swing around a certain magnetic-axis position is found to be very effective in changing the fieldline distribution pattern drastically, and thus dispersing the heat load.
\end{abstract}

(C) 2007 The Japan Society of Plasma Science and Nuclear Fusion Research

Keywords: LHD (Large Helical Device), helical divertor, long-pulse discharge, heat-load dispersion, real-time magnetic-axis swing, field-line tracing calculation

DOI: $10.1585 /$ pfr.2.043

\section{Introduction}

Sustaining steady-state, high-performance plasmas are crucial for realizing energy from a controlled magnetic nuclear-fusion reactor. In heliotron-type devices, the plasma-confining magnetic configuration is produced by external magnetic coils, thus, eliminating the need for plasma current. This simplifies steady-state operation and avoids dangerous plasma disruptions. Another advantage of the heliotron-type magnetic configuration is its intrinsic divertor magnetic configuration-the so-called helical divertor (HD) — which does not require additional coils for the divertor magnetic structure. However, plasma-wall interaction issues such as particle recycling and erosion of plasma-facing components during steady-state operation are common for experimental magnetic-fusion devices.

The Large Helical Device (LHD) is the largest superconducting heliotron-type device, with a set of $l=2$, $m=10$ helical coils and three pairs of poloidal coils. One of the major goals of the LHD is particle control with the HD for improving energy confinement and sustaining a high performance discharge with long duration time. Unlike the scrape-off layer in a tokamak poloidal divertor, the edge field-line structure outside the last closed flux surface (LCFS) in the HD configuration consists of a stochastic field-line layer, residual islands, and an edge-surface layer [1]. Furthermore, the structure varies significantly

author'se-mail: h-ogawa@nifs.ac.jp with different operational magnetic configurations, such as the major radius of the magnetic axis $\left(R_{\mathrm{ax}}\right)$. The heatand particle-deposition distributions on the HD plates are highly non-uniform, and strongly influenced by the edge field-line structure and hence by the operational magnetic configuration [2].

In the LHD, long-pulse discharge experiments have been conducted mainly using ICRF heating [3]. One of the limitations of long-pulse discharges is outgassing resulting from temperature rise of the divertor plate. The nonuniform heat deposition on the HD plate, mentioned above, causes a local rise of the plate temperatures [4]. To extend the discharge duration, the heat-handling capability of the divertor plate has been improved [5]. In addition, during experiments in 2004, a new operational scenario that disperses the divertor heat load was developed. The new scenario uses $R_{\mathrm{ax}}$ swing during the discharge, and as a result, a discharge longer than 54 minutes with $1.6 \mathrm{GJ}$ of total input energy was sustained [6].

This paper describes the investigation of the relationship between the heat and particle deposition on the HD plate and the edge magnetic structure. We trace many field lines from outside of the LCFS to the divertor plate to find the magnetic structure in the edge-plasma region. The connection length of the field lines, $L_{\mathrm{c}}$, is defined as the distance along a field line between the trace starting point and the striking point on the divertor plate. We found that a 
majority of the heat and particle fluxes flow along field lines with a long connection length-typically over several hundred meters. Those long field lines connect the LCFS and the divertor plates [2]. This has led to using $R_{\mathrm{ax}}$ swing operation for reduction of the heat load during long-pulse operation.

\section{Experimental and Numerical Set- up}

In LHD, four antennas for ICRF heating are installed on the top and bottom ports in toroidal sections 3 and 7 [3]. For long-pulse discharges, with duration over 10 minutes, the total ICRF heating input power was less than $1 \mathrm{MW}$ at 38.47 MHz. The working gas is helium, and the frequency was determined by the cyclotron-resonance condition of the minority ions $\left(\mathrm{H}^{+}\right.$in this case $)$.

Magnetic-axis position $\left(R_{\mathrm{ax}}\right)$ swing operation during long-pulse discharges is conducted by sweeping the currents of three pairs of poloidal field coils, while keeping the helical coil currents constant. The $R_{\mathrm{ax}}$ swing rate is limited to $0.5 \mathrm{~mm} / \mathrm{s}$, because of the temperature rise of the superconducting coils resulting from the induction current.

Figure 1 (a) shows a schematic view of the divertor plate arrays in the LHD. Divertor plates are arranged along the helical coil cans, as shown in Fig. 1 (b). In LHD, there are 10 sets of such arrays. Divertor plates (typically $190 \times 90 \times 15 \mathrm{~mm}$ ) consist of isotropic carbon, and cooled by water [7]. Thermocouples are embedded in all toroidal sections of the divertor plates, in horizontally and vertically elongated poloidal cross-sections, as shown in Fig. 1 (c), to measure temperature rises. The thermocouples are located at the center of the divertor plates, and at a $10 \mathrm{~mm}$ depth from the surface. The sampling time of the thermocouple data is $1 \mathrm{~s}$. Three Langmuir probe arrays are installed in the torus inboard side (LP\#1 and 2) and bottom (LP\#3) divertor plates, as shown in Fig. 1 (b), to measure the particle-flux profiles on the divertor plates. The dome-type electrodes (diameter $=2 \mathrm{~mm}$ ) of the Langmuir probe arrays are located along the edge of the divertor plates, with a $6 \mathrm{~mm}$ spacing.

A field-line tracing calculation was conducted using LHD lines-of-force code [8] and KMAG code [9] under vacuum conditions to investigate the relationship between the magnetic structure and heat- and particle-deposition distributions on the HD. Using KMAG code, the crossfield transport is simulated by a random-walk in the fieldline tracing. At every step along the field line, $\lambda$, the position of the field line deviates by $\delta$ in the plane perpendicular to the field lines, with random azimuthal directions [10]. The random process smoothes the fine field-line structure. The level of randomness, $\delta^{2} / \lambda$, roughly equals the observed level of a typical tokamak experiment. In this model, the thickness $\left(\Delta_{\text {div }}\right)$ of the divertor channel, or scrape-off layer, is given by $\delta\left(L_{\mathrm{c}} / \lambda\right)^{1 / 2}$. Our selection of typical parameters $(\delta=0.6 \mathrm{~mm}, \lambda=0.2 \mathrm{~m})$ is (a)

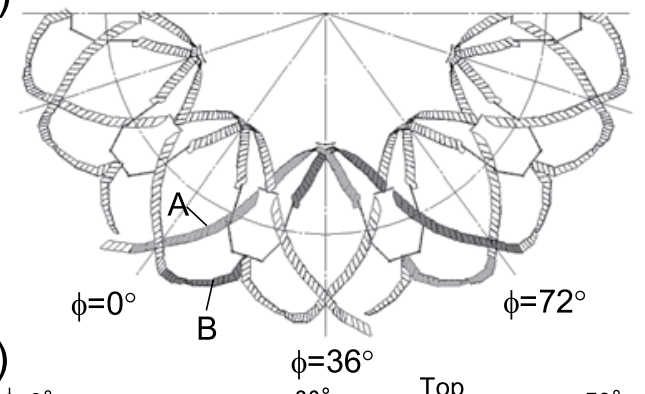

(b)

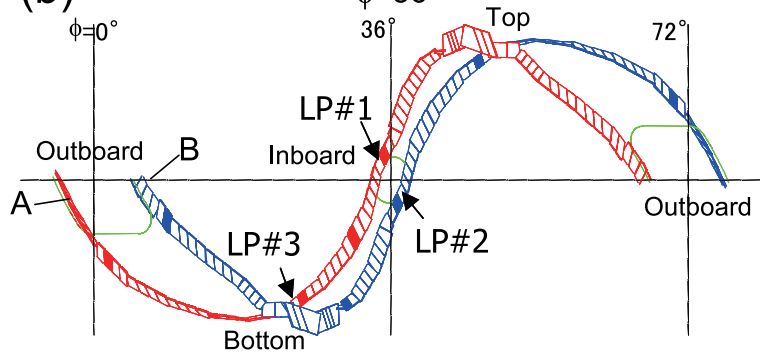

(c)

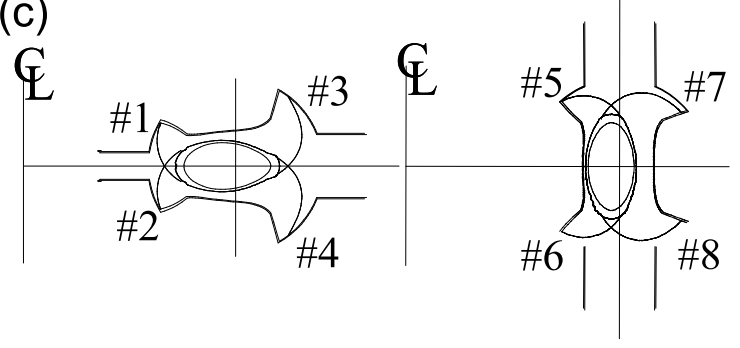

Fig. 1 (a) Schematic view of the divertor-plate array in the LHD for half of the torus. (b) Enlarged view of the divertorplate array labeled "A" and "B" in (a). Positions of the Langmuir probe arrays are shown. (c) Positions of the thermocouples embedded in the divertor plates in horizontally and vertically elongated cross-sections.

consistent with typical divertor experiments $\left(\Delta_{\mathrm{div}} \sim 6 \mathrm{~mm}\right.$, $\left.L_{\mathrm{c}} \sim 20 \mathrm{~m}\right)$.

In this study, field-line calculations are done under vacuum conditions, that is, without effects of finite beta and toroidal-plasma current flowing through the plasma. With increasing plasma beta, Pfirsch-Schluter current increases, changing the magnetic-field structure. As described in reference [11], changes in the field-line distribution resulting from beta are small for beta less than $1 \%$. The beta of the discharge in this study is less than $0.5 \%$. Toroidal current also influences the magnetic structure. In the discharges presented in this paper, plasma current is typically $20 \mathrm{kA}$ at $B=2.5 \mathrm{~T}$. Assuming this level of plasma current flows at the magnetic axis, the shift of the distribution on the divertor plate at the inner side of the torus is estimated to be $2 \mathrm{~mm}$, and is smaller than the spatial resolution $(6 \mathrm{~mm})$ of the Langmuir probe array. Thus, the effects of toroidal current and plasma beta are negligible in this experiment. 


\section{Results and Discussion}

\subsection{Heat- and particle-deposition profiles on the divertor plates during HD discharges}

A comparison between the measured heat- and particle-deposition distribution on the divertor plates, and the distribution of the strike points of the field lines from the main plasma region, obtained by the KMAG code including a random-walk process $(\lambda=0.2 \mathrm{~m}, \delta=0.6 \mathrm{~mm})$, was done for typical inward $\left(R_{\mathrm{ax}}=3.60 \mathrm{~m}\right)$ and outward $\left(R_{\mathrm{ax}}=3.75 \mathrm{~m}\right)$ shifted magnetic-axis configurations. Two thousand starting points were distributed just inside the LCFS in this calculation. The measured heat- and particledeposition profiles were consistent with the distribution of the field lines striking points on the HD plates [2].

The present study conducted a comparison between the measured heat-deposition profiles and the striking points distribution of field lines with long connection lengths for various values of $R_{\mathrm{ax}}\left(R_{\mathrm{ax}}=3.60,3.70,3.90\right.$, $4.00 \mathrm{~m}$ ) during NBI heated discharges. In Fig. 2 (a), the temperature rise, $\Delta T$, at the thermocouple-embedded divertor plates (Div\#1-8 in Fig. 1 (c)) is plotted as a function of the total deposited energy on the divertor plate in the $R_{\mathrm{ax}}=3.6 \mathrm{~m}$ configuration. The total deposited energy on the divertor, $E_{\mathrm{div}}$, is defined as:

$$
E_{\mathrm{div}}=\int_{t=0}^{t}\left(P_{\mathrm{abs}}-P_{\mathrm{rad}}\right) \mathrm{d} t,
$$

where $P_{\text {abs }}$ and $P_{\text {rad }}$ are the absorbed NB power and the radiation power, respectively. In this figure, $\Delta T$ for Div\#1-8 show linear dependences on $E_{\text {div }}$ without saturation. The heat load on these divertor plates can be expressed well by the normalized temperature rise, $\Delta T / E_{\text {div }}$. Figure 2 (b) shows the $\Delta T / E_{\mathrm{div}}$ at the plate with various poloidal locations (Div\#1-8, Fig. 1 (c)). Their toroidal locations differ, but we confirmed that temperature increases at the plate are insensitive to the toroidal location in this experiment. Figure 2(b) shows the relationship between the normalized $\Delta T$ and the position of the divertor plates for various values of $R_{\mathrm{ax}}$. For $R_{\mathrm{ax}}=3.60 \mathrm{~m}, \Delta T / E_{\mathrm{div}}$ at Div\#1 and 2 is larger than at other divertor plates, indicating that the heat deposition on the torus inboard-side divertor plates is dominant in this configuration-this is consistent with the previous study [2]. For outward-shifted cases, the normalized temperature rise at Div\#5 and 6 exceeds that at Div\#1 and 2 . These experimental results clearly show that heat deposition on the HD plate is highly localized. Figure 2 (c) shows the results of LHD lines-of-force code calculations. The height of the column is the ratio of the number of field lines connecting to Div\#1-8 to the total number of tracedfield lines with connection lengths over 10 toroidal turns ( $\sim 250 \mathrm{~m})$. Note that such long field lines connect the LCFS to the divertor plates, and are considered the main channel for the heat and particle transport [2]. The distribution of the field lines with long connection lengths on the HD plate is also localized, as shown in Fig. 2 (c). For $R_{\mathrm{ax}}=3.60 \mathrm{~m}$, the ratio of the long field-line connections is large at Div\#1 (a)

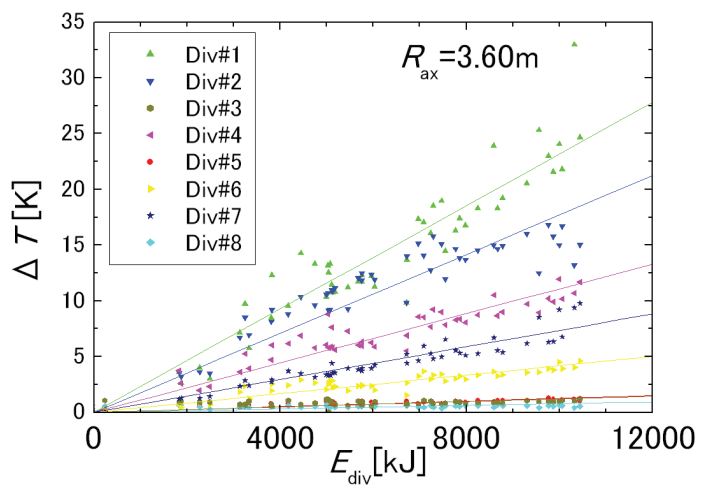

(b)

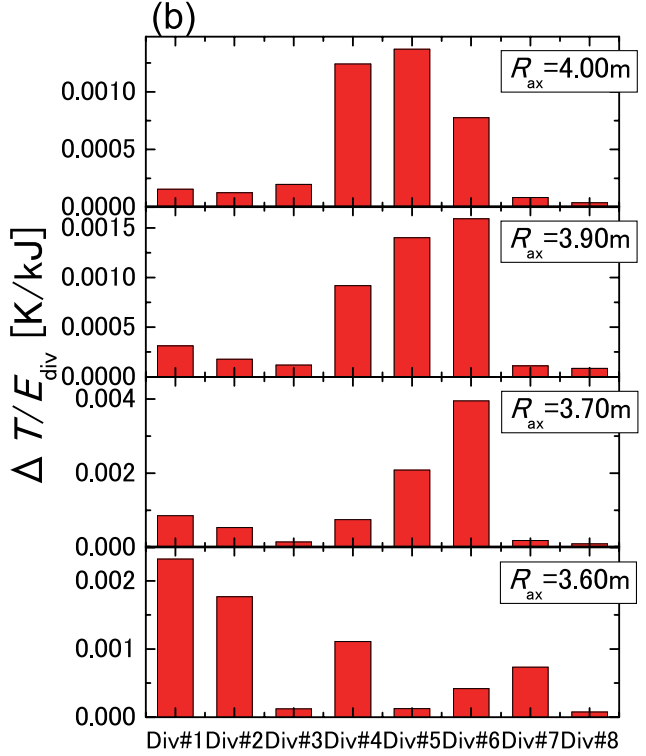

(c)

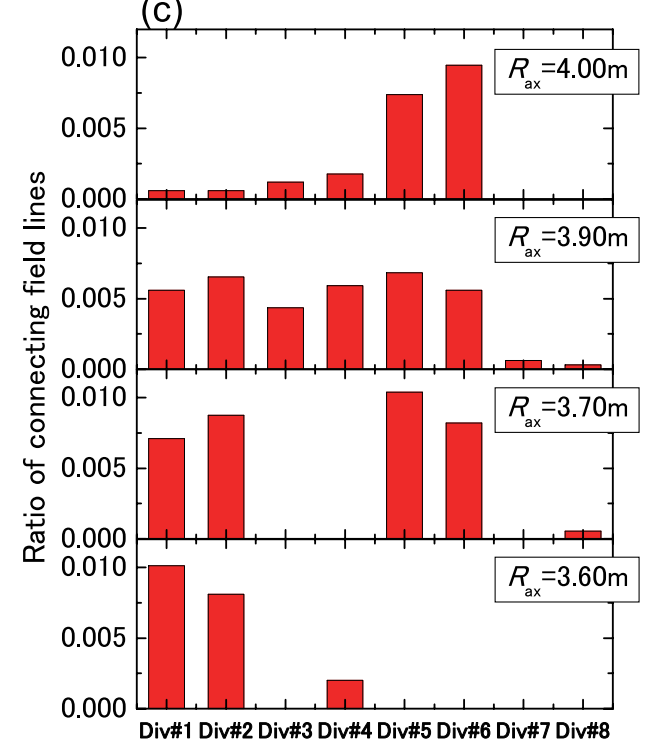

Fig. 2 (a) $E_{\text {div }}$ dependence of $\Delta T$ for Div\#1-8 divertor plates in NBI-heated discharges under the $R_{\mathrm{ax}}=3.60 \mathrm{~m}$ configuration. (b) $\Delta T$ is almost proportional to $E_{\text {div }}$. Thus, the normalized temperature, $\Delta T / E_{\text {div }}$ for Div\#1-8 is shown for four different $R_{\mathrm{ax}}$. (c) The ratio of the long field lines connecting to Div\#1-8 to the total number of traced-field lines for four different $R_{\mathrm{ax}}$. 


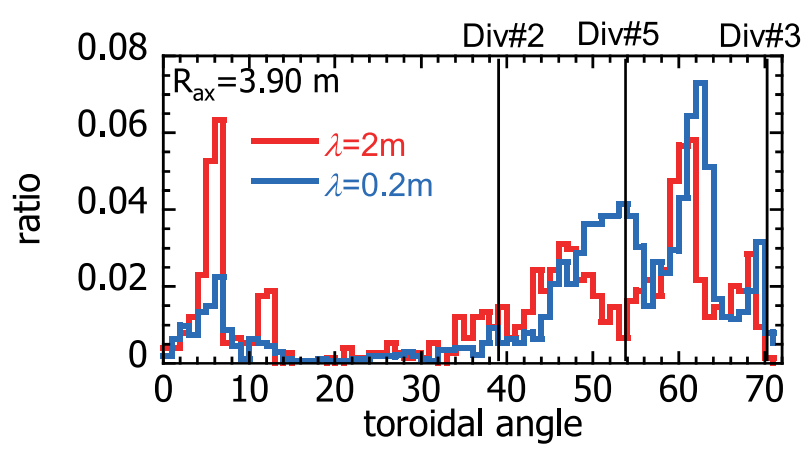

Fig. 3 The profiles of the ratio of the field-line deposition on the divertor plates to the total number of traced-field lines along divertor plate array "B" in Fig. 1, which are calculated with KMAG code for two different random-walk parameters, $\lambda(=2,0.2 \mathrm{~m})$, with fixed $\delta(=0.6 \mathrm{~mm})$.

and 2, and this corresponds to the large heat depositions on these plates, shown in Fig. 2 (b). As $R_{\mathrm{ax}}$ shifts outward, the ratio becomes large at Div\#5 and 6, and this also corresponds to the large heat deposition on these plates. For $R_{\mathrm{ax}}=3.70$ and $3.90 \mathrm{~m}$, on the other hand, the ratios at Div\#1 and 2 are not small compared to those at Div\#5 and 6 , even though there is little heat deposition at Div\#1 and 2. These results suggest that the heat-deposition profile on the HD plates is not determined solely by the distribution of the long field lines. Figure 3 shows the ratio of the number of long field lines connected to the divertor plates, to the total number of long field lines along divertor plate array " $\mathrm{B}$ " in Fig. 1, which is calculated using the KMAG code for two different random-walk parameters, $\lambda$, with fixed $\delta$ ( $=0.6 \mathrm{~mm})$. The longer $\lambda$ corresponds to lower diffusivity. The poloidal positions of the thermocouples (Div\#2, 3, 5) are indicated in Fig. 3. For longer $\lambda(\lambda=2 \mathrm{~m})$, the ratios at these divertor-plate positions (Div\#2 (39 degree), 5 (54 degree), 3 (70 degree) are about the same. This is also seen in Fig. 2 (c) for $R_{\mathrm{ax}}=3.90 \mathrm{~m}$ and $\lambda=+\infty \mathrm{m}$. However, for $\lambda=0.2 \mathrm{~m}$ (higher diffusivity), the ratio at Div\#5 is significantly higher than those at Div\#2 and 3. This is similar to the observed heat distribution for $R_{\mathrm{ax}}=3.90$, as shown in Fig. 2 (b). This result demonstrates the effect of the crossfield transport on the determination of the heat-deposition profile on the HD plates, in addition to a broadening of the particle-flow channel.

\subsection{Operation of magnetic-axis swing for re- duction of the heat load on the divertor plates}

From the point of view of heat removal at the divertor, a uniform heat-deposition distribution is desirable. To find the $R_{\mathrm{ax}}$ configuration with a more uniform heatdeposition distribution on the HD plate, a investigation of the heat- and particle-deposition profiles was conducted for $R_{\mathrm{ax}}=3.60 \mathrm{~m}, 3.65 \mathrm{~m}, 3.68 \mathrm{~m}, 3.69 \mathrm{~m}, 3.70 \mathrm{~m}$ and $3.75 \mathrm{~m}$. Figure 4 shows the $\Delta T$ and the sum of the ion-saturation
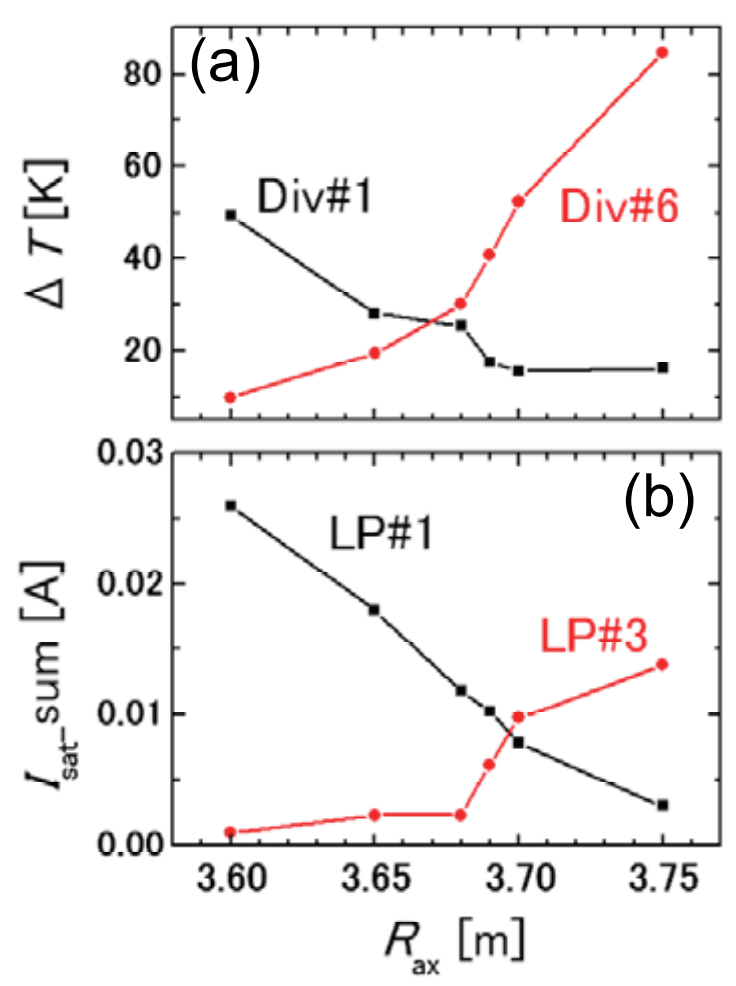

Fig. $4 R_{\mathrm{ax}}$ dependences of (a) the temperature rises, $\Delta T$, at Div\#1 (black) and 6 (red), and (b) the sum of the ionsaturation currents to the Langmuir probe arrays, LP\#1 (black) and 3 (red). In this case, $\Delta T$ is the temperature increase during a discharge.

currents to the Langmuir probe arrays at the torus inboardside and bottom side. With an increase of $R_{\mathrm{ax}}$, the heat and particle deposition at the torus inboard-side (Div\#1 and LP\#1) decreases, and increases at the bottom (Div\#6 and LP\#3) and at the two curves that cross around $R_{\mathrm{ax}}=3.67-$ $3.70 \mathrm{~m}$ in Fig. 4 (a) and (b). Therefore, the heat and particle deposition appears to be more uniform at $R_{\mathrm{ax}}=3.67$ $3.70 \mathrm{~m}$. Figure 5 shows the profiles of the proportion of the connected long-field lines along divertor-plate array " $\mathrm{B}$ " (see Fig. 1) for various $R_{\mathrm{ax}}$ configurations. All of these profiles exhibit spikes, and the spike position changes with a change in $R_{\mathrm{ax}}$. There are two distribution patterns. For inward-shifted cases $\left(R_{\mathrm{ax}}=3.60 \mathrm{~m}\right)$, the depositions occur between 5 and 40 degrees of the toroidal angle (at the torus inboard side). For outward-shifted cases $\left(R_{\mathrm{ax}}=3.70\right.$, $3.75 \mathrm{~m}$ ), deposition is localized around 50 degrees (at the torus top side). When the configuration changes from large to small $R_{\mathrm{ax}}$, the field-line distribution pattern alters rapidly at $R_{\mathrm{ax}}=3.66 \mathrm{~m}$. Thus, even a small swing around $R_{\mathrm{ax}}=3.66$ should be effective in dispersing the heat load. This is consistent with the experiment described below, where swing operation in the range of $R_{\mathrm{ax}}=3.65-3.69 \mathrm{~m}$ proved effective.

As shown in the previous section, the heat-deposition profile on the HD is localized, and the localized-heat load causes a large increase in the temperature of certain diver- 


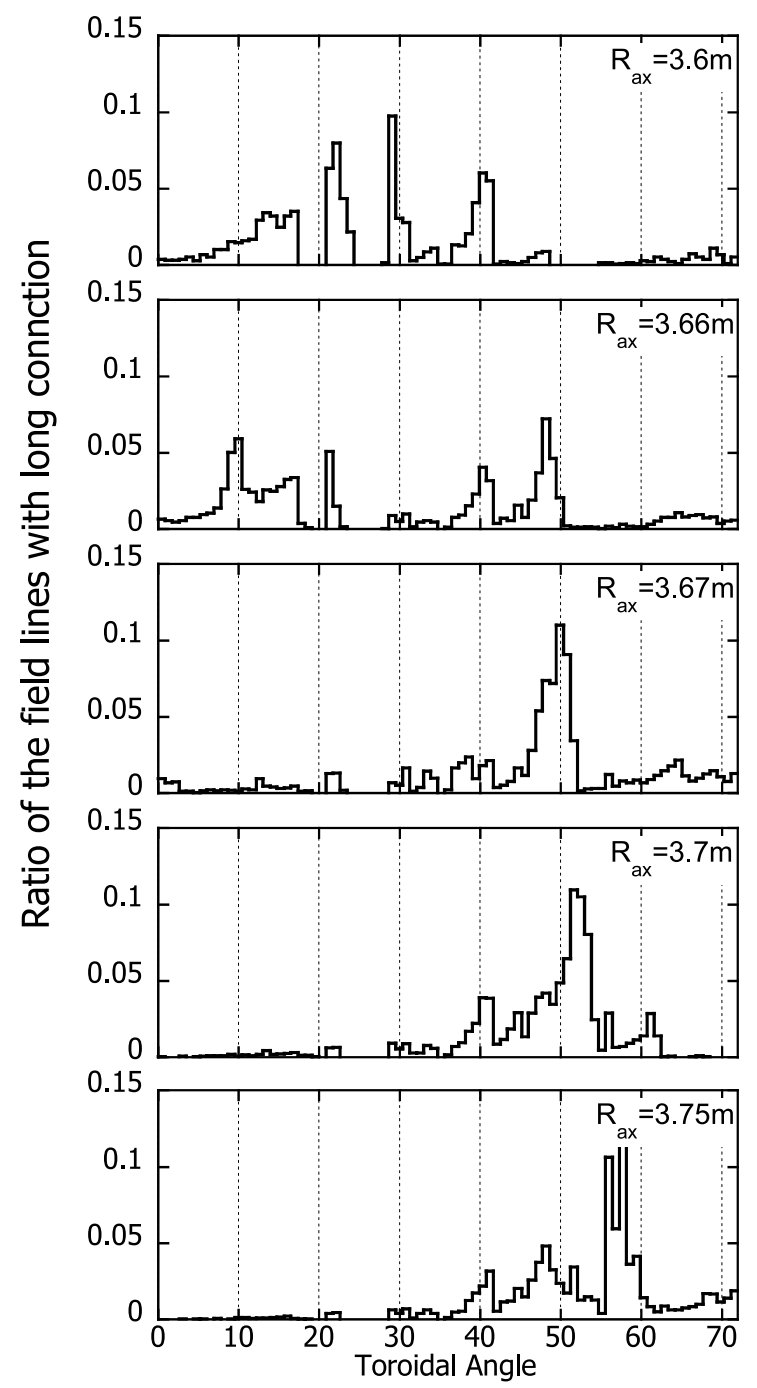

Fig. 5 The profiles of the ratio of the long field line connections (over 10 toroidal turns) along divertor plate array "B" in Fig. 1 for various $R_{\mathrm{ax}}$ configurations. These profiles are calculated by LHD lines-of-force code.

tor plates during long-pulse discharges. As a result, the discharges are terminated by uncontrollable increases in electron density, radiation collapse resulting from outgassing, or impurity release from the divertor plates. To disperse the heat load on the divertor plates, $R_{\mathrm{ax}}$ swing operation has been proposed and conducted.

The heat- and particle-deposition distributions on the HD plate during $R_{\mathrm{ax}}$ swing operation were investigated to find a suitable range of the swing. Figure 6 (a) shows the time evolutions of $\Delta T$ and $\mathrm{d} T / \mathrm{d} t$ at the torus inboard-side (Div\#1) and bottom (Div\#6) divertor plates during $R_{\mathrm{ax}}$ swing operation in the range of $R_{\mathrm{ax}}=3.62-3.65 \mathrm{~m}$. In this case, $\Delta T$ increases monotonically at both divertor plates, and there is no apparent effect of the swing in $\Delta T$. Figure 6(b) also shows the waveforms for $\Delta T$ and $\mathrm{d} T / \mathrm{d} t$ during swing operation in the range of $R_{\mathrm{ax}}=3.65-3.69 \mathrm{~m}$. In this case, modulation of $\Delta T$ by the swing clearly appears on Div\#1 and 6 and is caused by movement of the localized
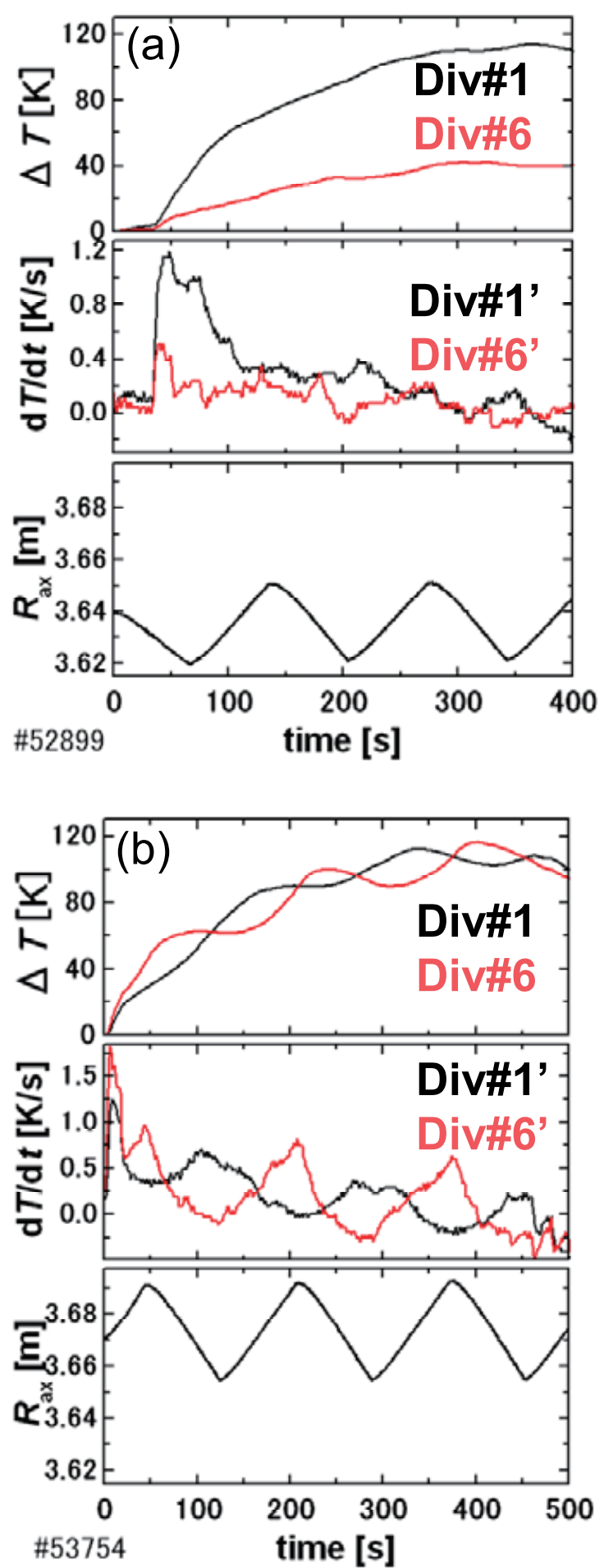

Fig. 6 Temperature increase, $\Delta T$, and rate of temperature change, $\mathrm{d} T / \mathrm{d} t$, at torus inboard-side (Div\#1) and bottom (Div\#6) divertor plates during $R_{\mathrm{ax}}$ swing operation in the range of (a) $R_{\mathrm{ax}}=3.62-3.65 \mathrm{~m}$ and (b) $R_{\mathrm{ax}}=3.655$ $3.69 \mathrm{~m}$.

heat-deposition area with $R_{\mathrm{ax}}$ swing, as shown in Fig. 5 .

The time evolutions of the ion-saturation currents, $I_{\text {sat }}$, to the torus inboard-side (LP\#1) and bottom (LP\#3) Langmuir probe arrays are depicted in Fig. 7 (a). These show that the particle flux to the divertor plate varies with $R_{\mathrm{ax}}$ 
(a)

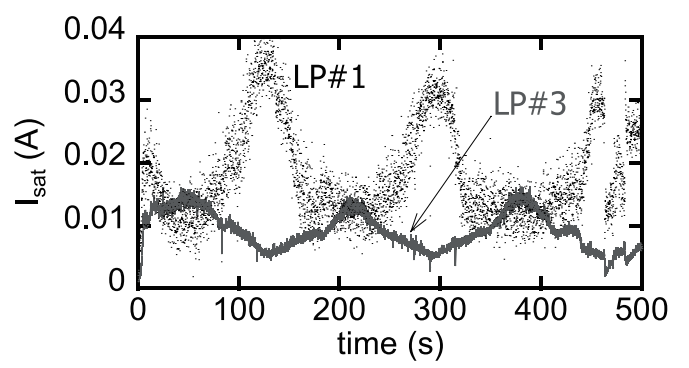

(b)

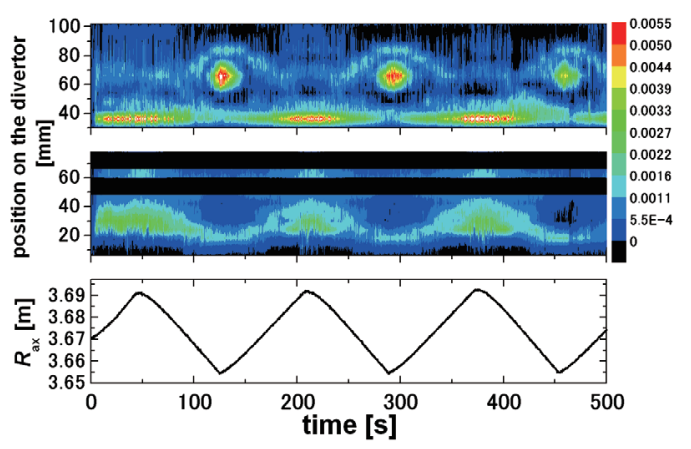

(c)

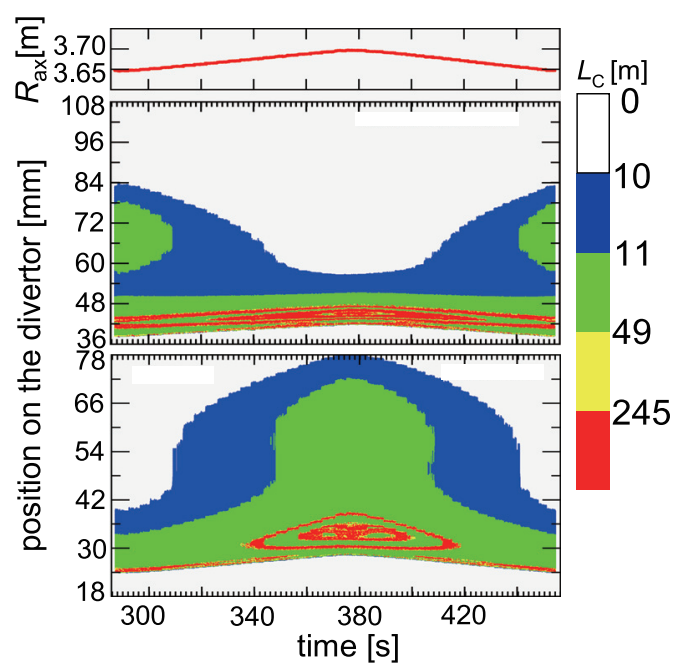

Fig. 7 (a) Time evolutions of the ion-saturation currents, $I_{\text {sat }}$, to the torus inboard side (LP\#1) and bottom (LP\#3) Langmuir probe arrays during the same discharge as shown in Fig. 6(b). (b) Time evolutions of the ion-saturation current profiles on the torus inboard side (top) and bottom (middle) divertor plates are shown during $R_{\mathrm{ax}}$ swing. This discharge is the same as that shown in Fig. 6(b). (c) Time evolutions of the profiles of the field-line connection lengths, $L_{\mathrm{c}}$, on the torus inboard-side (middle) and bottom (bottom) divertor plates during a cycle of the same $R_{\mathrm{ax}}$ swing as shown in Fig. 6 (b), calculated by LHD lines-of-force code. swing. Figure 7 (b) shows the time evolutions of the $I_{\text {sat }}$ profiles on LP\#1 and 3, during $R_{\mathrm{ax}}$ swing. The $I_{\text {sat }}$ profiles change with the $R_{\mathrm{ax}}$ swing, and thus the particle-load concentration to a specific area on the divertor plates can be avoided with $R_{\mathrm{ax}}$ swing operation. Figure 7 (c) depicts the time evolutions of the profiles of connection lengths, $L_{\mathrm{c}}$, of various ranges, on divertor plates LP\#1 and 3. They are calculated by the LHD lines-of-force code, and the starting points for the field line tracing are on the divertor plates along the Langmuir probe arrays for a $R_{\mathrm{ax}}$ swing cycle. Comparing the $I_{\text {sat }}$ profiles in Fig. 7 (b) with the $L_{\mathrm{c}}$ profiles in Fig. 7 (c), we find that the $I_{\text {sat }}$ profiles on both divertor plates are similar in shape to the $L_{\mathrm{c}}$ profiles, indicating that a majority of the particles flow outwards along the field lines with long connection lengths.

\section{Conclusion}

The heat- and particle-deposition distributions on the divertor plates in the HD have been investigated both experimentally and numerically. Their localized-deposition distributions have been observed by thermocouples and Langmuir probe arrays, embedded in the divertor plates. The localized distributions of field lines on the divertor plates with long connection lengths have been calculated by field-line tracing calculations for various $R_{\mathrm{ax}}$ configurations. Comparison of the heat- and particle-distribution profiles observed in the experiments, and the distribution of field lines, which connect the divertor plates and the LCFS, reveals that the heat- and particle-deposition profiles are strongly correlated with the field lines with long connection lengths. Field-line tracing with a random-walk process shows that the cross-field transport also affects the heat- and particle-deposition profiles. When $R_{\mathrm{ax}}$ of the configuration is changed, a sudden change in the long fieldline distribution pattern occurs at $R_{\mathrm{ax}}=3.66-3.67 \mathrm{~m}$. A swing around such a location is very effective in dispersing the heat load. This investigation suggests using $R_{\mathrm{ax}}$ swing operation to disperse the heat- and particle-deposition distribution on the HD plates during long-pulse discharges.

\section{Acknowledgement}

The authors thank the technical staff of the LHD group for their continuous support. This work was supported by NIFS budgets NIFS06ULPP511 and NIFS06GLPP004.

[1] N. Ohyabu et al., Nucl. Fusion 34, 387 (1994).

[2] S. Masuzaki et al., Nucl. Fusion 42, 750 (2002).

[3] R. Kumazawa et al., Nucl. Fusion 46, S13 (2006).

[4] M. Shoji et al., J. Nucl. Mater. 337-339, 186 (2005).

[5] Y. Kubota et al., Fusion Eng. Des. 75-79, 297 (2005).

[6] K. Saito et al., J. Nucl. Mater. 363-365, 1323 (2007).

[7] S. Masuzaki et al., J. Nucl. Mater. 290-293, 12 (2001).

[8] T. Watanabe et al., Nucl. Fusion 46, 291 (2006).

[9] Y. Nakamura et al., J. Plasma Fusion Res. 69, 41 (1993).

[10] T. Morisaki et al., Contrib. Plasma Phys. 42, 321 (2002).

[11] S. Masuzaki et al., Fusion Sci. Technol. 50, 361 (2006). 